\title{
Dielectric relaxation of polychlorinated biphenyl/toluene mixtures: Component dynamics
}

\author{
Daniele Cangialosi, ${ }^{1, a)}$ Angel Alegría, ${ }^{1,2}$ and Juan Colmenero, ${ }^{1,2,3}$ \\ ${ }^{1}$ Centro de Física de Materiales Centro Mixto (CSIC-UPV/EHU), Facultad de Química, Apartado 1072, \\ 20080 San Sebastián, Spain \\ ${ }^{2}$ Departamento de Física de Materiales, Universidad del País Vasco (UPV/EHU) Apartado 1072, \\ 20080 San Sebastián, Spain \\ ${ }^{3}$ Donostia International Physics Center, Paseo Manuel de Lardizabal 4, 20018 San Sebastián, Spain
}

(Received 22 February 2008; accepted 6 May 2008; published online 11 June 2008)

\begin{abstract}
The dynamics of homogenous polychlorinated biphenyl (PCB54)/toluene mixtures have been investigated by means of broadband dielectric spectroscopy. The mixture presents dynamical heterogeneity, alike miscible polymer blends, which is manifested with the presence of two relaxational processes. The relatively slow one has been attributed to the motion of PCB54 in the mixture, whereas the relatively fast one was related to the toluene dynamics in the mixture. These results have been interpreted according to the self-concentration concept, first introduced to describe the dynamics of miscible polymer blends, which relies on the limited size of the cooperative length scale in glass-forming liquids. The self-concentration concept has been incorporated in the AdamGibbs theory of the glass transition relating the characteristic relaxation time and the length scale for structural relaxation to the configurational entropy. This allowed the determination of the cooperative length scale of PCB54 and toluene both in mixture and alone through the fitting of a single parameter, namely, that connecting the cooperative length scale to the configurational entropy. This length scale resulted to be in the range of 1-2 nm for all systems. Finally, the out-of-equilibrium dynamics of toluene induced by the selective freezing in of PCB54 has been examined and its relation to the Johari-Goldstein relaxation of pure toluene was critically discussed. () 2008 American Institute of Physics. [DOI: 10.1063/1.2937449]
\end{abstract}

\section{INTRODUCTION}

The occurrence of the glass transition in supercooled liquids is one of the main unsolved problems in condensed matter physics and huge amount of work has been devoted to the understanding of this phenomenon. ${ }^{1}$ In an attempt to get new insights about the nature of the glass transition, the vast majority of the research in this field has concerned the study of single component glass formers, whereas only in recent years increasing attention has been paid to the study of bicomponent glass-forming systems.

As a subclass of these systems, that of miscible polymer blends has been one of the most investigated. This is due to the peculiar relaxational pattern of these systems displaying dynamical heterogeneity, which is manifested with the presence of two dynamics related to the glass transition phenomenon. This phenomenology has been recently explained invoking the concept of "self-concentration" that was first introduced by Chung and Kornfield ${ }^{2}$ and later was developed by Lodge and McLeish. ${ }^{3}$ This can be explained as follows: due to the relatively localized nature of the structural dynamics (the $\alpha$ relaxation associated with the glass transition), the effective concentration of one component in the volume relevant for its segmental relaxation is always larger than that of the macroscopic one. Due to the fact that the volume probed by selective techniques can be centered on monomers

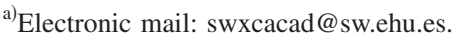

of each component type, two segmental dynamics usually show up in the relaxation pattern of the blend.

Self-concentration effects are now well established to describe the dynamics of miscible polymer blends ${ }^{3-18}$ and diblock copolymers, ${ }^{9}$ even nanophase segregated ones. ${ }^{19}$ A recent review critically scrutinizes the application of the selfconcentration concept into the dynamics of miscible polymer blends. ${ }^{20}$ For these systems, the presence of chain connectivity induces an effective concentration markedly larger than the macroscopic one. However, by investigating the dynamics of a polychlorinated biphenyl (PCB54) diluted in 1,4-polybutadiene (PB), it has been recently shown that selfconcentration effects, although to a lower extent compared to polymer blends, are also important for low molecular weight glass formers. ${ }^{21}$ Therefore, although the self-concentration concept has been introduced for miscible polymer blends, this can be reasonably extended to low molecular weight mixtures. The self-concentration concept can be rationalized considering that the characteristic length scale for the glass transition (the so-called cooperative length scale) seems to extend over the first shell surrounding the structural unit under consideration, as recently highlighted in both polymeric ${ }^{22}$ and low molecular weight glass formers. ${ }^{21}$ In the case of PCB54 in diluted mixtures with PB, this means that, although the values of the macroscopic concentration practically are equal to zero $(\phi=0)$, the resulting value of the effective concentration is of the order of $\phi_{\mathrm{eff}} \approx 0.1$ at the glass transition temperature $\left(T_{g}\right)$. This means that about ten 
basic structural units are involved in the cooperative volume: 1 of PCB54 and the rest composed by PB structural units.

Starting from these premises, in this work, we have investigated the structural dynamics of PCB54/toluene system, a mixture displaying large dynamical asymmetrybeing the glass transition temperature $\left(T_{g}\right)$ of the two components of the mixtures very different $\left(T_{g}^{\mathrm{PCB} 54}=246 \mathrm{~K}\right.$ and $T_{g}^{\mathrm{tol}}=117 \mathrm{~K}$ ) - by means of broadband dielectric spectroscopy (BDS). We show that this system displays two dynamics, a feature already observed in other bicomponent mixtures displaying relatively large dynamical contrast. ${ }^{23-26} \mathrm{We}$ interpret these results considering that the effective concentration in the characteristic volume for the relaxation is different from the average one and describe these results in the framework of a model that was presented by us for polymer blends ${ }^{11,27-29}$ and recently extended to low molecular weight glass formers, ${ }^{21}$ incorporating the self-concentration concept in the Adam-Gibbs theory ${ }^{30}$ of the glass transition (AG-SC model).

The central parameter of the Adam-Gibbs theory is the configurational entropy. In particular, according to this theory, the decrease in this quantity with decreasing temperature induces a slowing down of the dynamics and an increase in the cooperative length scale for the structural relaxation. The AG-SC model relies on the fitting of a single parameter, namely, that connecting the cooperative length scale to the configurational entropy. This means that the model is able to provide the characteristic length scale for the glass transition. To confirm the reliability of AG-SC model in providing this length scale, we remark that this has been independently determined for PCB54 diluted in polybutadiene through simple geometric arguments based on the change with the concentration of the distance between PCB54 molecules. ${ }^{21}$ The soobtained length scale has been found to be in excellent agreement with that obtained within the AG-SC framework despite the assumptions made in this model.

Apart from the description of the equilibrium relaxation data of both PCB54 and toluene related dynamics through the AG-SC model, we discuss relaxation data of toluene at relatively low temperatures, where the out-of-equilibrium dynamics induced by the presence of rigid PCB54 induces a peculiar relaxation pattern arising from the restricted motion of toluene molecules. We discuss the relation of this motion to the secondary relaxation of toluene.

\section{EXPERIMENT}

The polychlorinated biphenyl was Monsanto's Aroclor 1254 (PCB54) with 54 wt \% chlorine content. The chlorine content corresponds to four to five chlorine atoms per PCB54 molecule, attached to the two condensed aromatic hydrocarbons that constitute the core of PCB54 molecules. This means that PCB54 is a rigid molecule. It was purchased from LGC Promochem and used as received. Toluene was purchased from Aldrich and was purified before use by employing the procedure followed by Schlaad and Müller ${ }^{31}$ to reach a purity $>99 \%$.

PCB54 and toluene are soluble at all concentrations and homogeneous PCB54/toluene solutions were prepared by

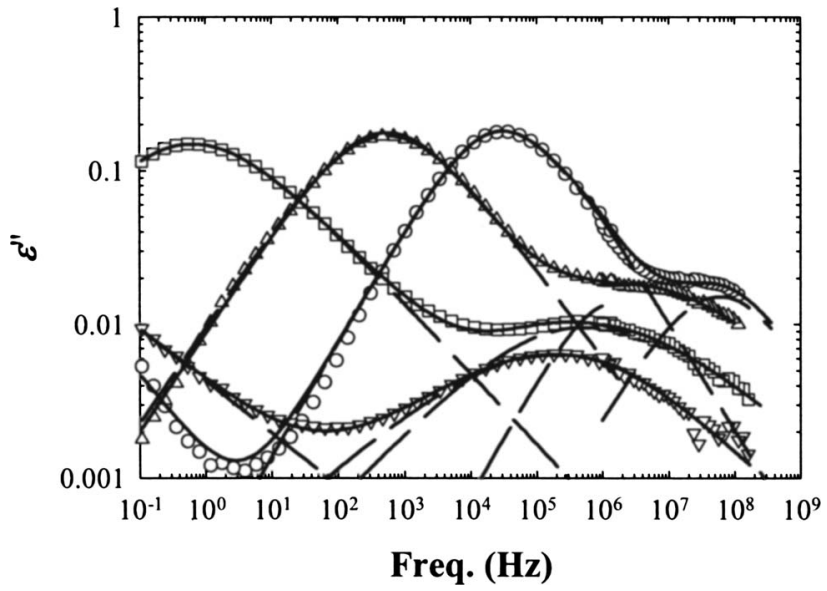

FIG. 1. Loss part of the dielectric permittivity vs frequency of PCB54/ toluene $35 / 65 \mathrm{wt} / \mathrm{wt} \%$ mixture at the following temperatures: $133 \mathrm{~K}$ (down triangles), $143 \mathrm{~K}$ (squares), $153 \mathrm{~K}$ (up triangles), and $163 \mathrm{~K}$ (circles).

adding excess toluene to PCB54. The solutions were subsequently poured on the bottom electrode of a liquid sample cell and the desired concentration was obtained via toluene evaporation. Teflon spacers were added to avoid short-circuit of the so-obtained dielectric capacitor. Solutions containing the following PCB54/toluene weight ratios were investigated: $70 / 30,50 / 50,35 / 65$, and $20 / 80 \mathrm{wt} / \mathrm{wt} \%$.

Dielectric measurements were carried out using two different equipments: a high precision dielectric analyzer (ALPHA, Novocontrol GmbH) over a wide frequency range $\left(3 \times 10^{-2}-3 \times 10^{6} \mathrm{~Hz}\right)$ was employed for all mixtures and an Agilent impedance analyzer HP4291B (frequency range of $10^{6}-10^{9} \mathrm{~Hz}$ ) was used for the mixtures with 35 and $20 \mathrm{wt} \%$ PCB54. A Novocontrol Quatro cryosystem for temperature control with a precision of $\pm 0.1 \mathrm{~K}$ was employed in all measurements. Homogeneous solutions were quenched in liquid nitrogen to avoid the possible crystallization of the mixtures. Measurements were performed in a wide temperature range in isothermal steps from the lowest temperature. Crystallization did not occur for any of the investigated mixtures as testified by the absence of those typical features showing up in dielectric spectra during the occurrence of this phenomenon (depression of the relaxation strength of the main relaxation time and appearance of a low frequency relaxational contribution). ${ }^{32}$

\section{RESULTS}

In Fig. 1, we present the permittivity loss versus frequency for PCB54/toluene mixture containing $35 \mathrm{wt} \%$ PCB54 at various temperatures. From the observation of the figure, it can be clearly recognized that two relaxational processes appear in the available frequency window: (i) a slow one with a relatively high dielectric strength and (ii) a fast (dielectrically weaker) one. These processes simultaneously show up in the available frequency range from 143 to $163 \mathrm{~K}$, whereas only the relatively fast one at $133 \mathrm{~K}$ is present. In this case, the high frequency tail of the relatively slow process is visible below $10^{2} \mathrm{~Hz}$.

Figure 2 displays the dielectric response of all investigated PCB54/toluene systems at different temperatures cho- 


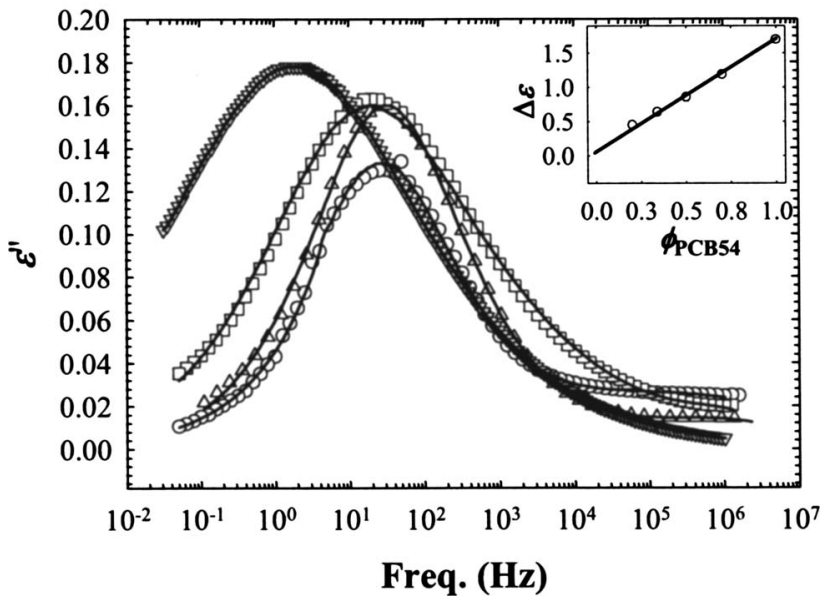

FIG. 2. Loss part of the dielectric permittivity vs frequency of PCB54/ toluene mixtures at the following concentrations of PCB54 and temperatures: $70 \%$ and $178 \mathrm{~K}$ (down triangles), $50 \%$ and $158 \mathrm{~K}$ (squares), 35\% and $148 \mathrm{~K}$ (up triangles), and $20 \%$ and $138 \mathrm{~K}$ (circles). (Inset) Dielectric strength of the low frequency process vs PCB54 concentration. The data for pure PCB54 and toluene are taken from Refs. 41 and 42, respectively.

sen in a way that the main relaxation peak shows up at about the same frequency, i.e., the (nearly) isochronal response. The following observations can be done from the analysis of the figure: (i) all mixtures present a relatively low frequency peak (at about $1-10 \mathrm{~Hz}$ ) with strength increasing with the PCB54 content; (ii) mixtures with 80 and $65 \mathrm{wt} \%$ toluene present a clear relaxational process at relatively high frequencies (about $10^{5}-10^{6} \mathrm{~Hz}$ ) (see also Fig. 1). This process, although present, cannot be reliably resolved in these conditions for mixtures with 50 and $30 \mathrm{wt} \%$ toluene; (iii) the low frequency peak of mixtures with 50 and $30 \mathrm{wt} \%$ toluene is more broadened in comparison with the peak showing up in the same frequency range for mixtures with a higher toluene content. The latter experimental evidence likely emerges from the presence of concentration fluctuations in bicomponent glass-forming systems ${ }^{23,33}$ and will not be the subject of further speculation in this work. On the other hand, the main focus of our investigation is the most probable relaxation time, namely, that associated with the maxima in permittivity loss versus frequency plots.

To assign each relaxational process present in PCB54/ toluene mixtures to specific molecular motions, it is worth noticing that the dielectric strength of the relatively slow process increases with the PCB54 content, whereas from the observation of Fig. 2, the relatively fast process increases its strength with the toluene content. ${ }^{34}$ Therefore, it is tempting to assign the slow and the fast processes to PCB54 and toluene related dynamics, respectively. The relaxation response of PCB54/toluene mixtures can be alternatively explained by hypothesizing that the peak with a larger relaxation strength manifesting at relatively low frequencies is related to the indistinguishable relaxation of PCB54 and toluene and the faster process is simply the secondary relaxation of toluene. This explanation would be supported by the fact that below $140 \mathrm{~K}$, the relaxation time of the latter process simply coincides with that of the pure toluene secondary relaxation for all mixtures. In this case, the change in the dynamics observed above 140-145 K for blends with large toluene con-

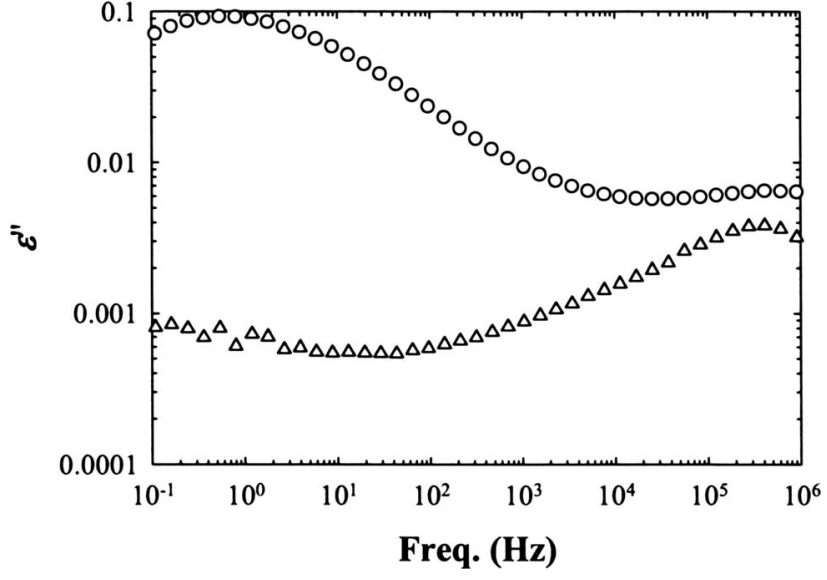

FIG. 3. Loss part of the dielectric permittivity vs frequency of PCB54/ toluene and OTP/toluene $35 / 65 \mathrm{wt} / \mathrm{wt} \%$ mixture at $143 \mathrm{~K}$

tent would agree with the results recently obtained by several authors, ${ }^{35-37}$ who actually observed a dynamic crossover for the secondary relaxation of several glass-forming liquids when passing through the $T_{g}$.

In order to unveil the real nature of the two relaxational processes present in PCB54/toluene mixtures, in the light of the two proposed hypotheses, we have investigated the dynamics of ortho-terphenyl/toluene mixture with $65 \mathrm{wt} \%$ ortho-terphenyl (OTP), a system dynamically analogous to PCB54/toluene mixture, as OTP's $T_{g}$ is very similar to that of PCB54 $\left(T_{g}=241 \mathrm{~K}\right)$. Contrary to PCB54, OTP has a barely measurable dielectric strength. ${ }^{38}$ Therefore, if part of the low frequency dielectric peak was attributable to some toluene related motions, that would be unveiled in OTP/ toluene systems, for which the very low dielectric activity of OTP does not interfere with the toluene dielectric response. This is shown in Fig. 3, where we have compared the permittivity loss versus frequency plots of OTP/toluene to that of PCB54/toluene mixtures with the same content of toluene (35 wt \%) at $143 \mathrm{~K}$. As can be observed, only a very low dielectric loss, with values around the limit of the equipment resolution, is present for OTP/toluene mixture in the frequency range where the low frequency relaxational contribution shows up for PCB54/toluene mixture. This means that no relaxational processes related to the dielectrically active toluene are present in this frequency window. On the other hand, the dielectric response of the two mixtures is practically identical at a relatively high frequency. Of course, one cannot exclude that at a relatively low temperature, where only Arrhenius-type temperature dependence is found for the high frequency process, toluene main relaxation process is hidden below PCB54 relaxation. In this case, the low frequency process can still be safely attributed to PCB54 dynamics, as this component possesses a relaxation strength consistently larger than that of toluene. All these observations indicate that the slow relaxational process displayed by PCB54/toluene can be fully attributed to the PCB54 related dynamics since toluene relaxation is well separated from that of PCB54 and is present at relatively higher frequencies.

To extract the relaxation time of each process present in PCB54/toluene mixtures, the dielectric spectra were fitted by 
a sum of Havriliak-Negami (HN) functions plus a direct current (dc) conductivity contribution. For the relative complex dielectric permittivity $\left(\varepsilon^{*}\right)$ in the frequency domain $(\omega)$, this reads $^{39}$

$$
\varepsilon^{*}(\omega)=-i \frac{\sigma_{\mathrm{dc}}}{\varepsilon_{0} \omega}+\varepsilon_{\infty}+\sum_{k=1}^{2} \frac{\Delta \varepsilon_{k}}{\left[1+\left(i \omega \tau_{\mathrm{HN}}^{k}\right)_{\mathrm{HN}}^{k}\right]_{\mathrm{HN}}^{k}},
$$

where $\tau_{\mathrm{HN}}$ is the $\mathrm{HN}$ relaxation time, $\varepsilon_{\infty}$ is the high frequency limit value of the dielectric constant, $\Delta \varepsilon$ is the dielectric strength of the relaxation process, $\alpha_{\mathrm{HN}}$ and $\gamma_{\mathrm{HN}}$ are the shape parameters of the HN function describing the symmetric and asymmetric broadening of the complex dielectric permittivity, respectively, $\sigma_{\mathrm{dc}}$ is the dc conductivity, and $\varepsilon_{0}$ is the vacuum permittivity.

A sum of two $\mathrm{HN}$ functions plus a de conductivity contribution, when needed, accurately describes the dielectric spectra of all studied mixtures, as shown in Figs. 1 and 2 by the solid lines. The dashed lines in Fig. 1 represent each contribution to the overall fits. The inset of Fig. 2 displays the dielectric strength of the peak showing up at relatively low frequencies. As can be observed, this varies between zero and the dielectric strength of pure PCB54 and depends linearly on the PCB54 concentration. This result provides an additional indication that the relatively slow relaxational process should be assigned to the PCB54 relaxation in the mixture.

The relaxation time corresponding to the permittivity loss maximum $\left(\tau_{\max }\right)$, taken as representative of the mean relaxation time, was evaluated from $\tau_{\mathrm{HN}}$ using the relation derived by Boersma et al., ${ }^{40}$

$$
\begin{aligned}
\tau_{\max }= & \tau_{\mathrm{HN}}\left[\left(\sin \frac{\alpha_{\mathrm{HN}} \pi}{2+2 \gamma_{\mathrm{HN}}}\right)^{1 / \alpha_{\mathrm{HN}}}\right. \\
& \left.\times\left(\sin \frac{\alpha_{\mathrm{HN}} \gamma_{\mathrm{HN}} \pi}{2+2 \gamma_{\mathrm{HN}}}\right)^{-1 / \alpha_{\mathrm{HN}}}\right]^{-1} .
\end{aligned}
$$

The so-obtained relaxation times are plotted in Fig. 4 versus the inverse of the temperature. The relaxation times of pure PCB54 and toluene, taken respectively from Refs. 41 and 42, are also displayed in Fig. 4. As a guide to better inspect the figure, the two relaxational processes displayed by each PCB54/toluene mixture, one related to PCB54 and the other to toluene, are displayed with the same color. From inspection of the figure, it can be concluded that the relatively slow process present in all PCB54/toluene mixtures speeds up with increasing toluene content. Furthermore, the temperature dependence of the relaxation time related to this process is non-Arrhenius for all investigated mixtures since the activation energy is larger the lower the temperature, as generally found for fragile glass formers. A similar pattern can be observed for the relatively fast component in PCB54/ toluene mixtures with a relatively high toluene content, namely, those containing 20 and 35 wt \% PCB54. However, in this case, the high activation energy is observed only for temperatures where the relaxation time is shorter than $10^{-7} \mathrm{~s}$. Similarly to the relatively slow component, this component is faster for the mixture with larger toluene content. However, at lower temperature (below about 140-145 K) this

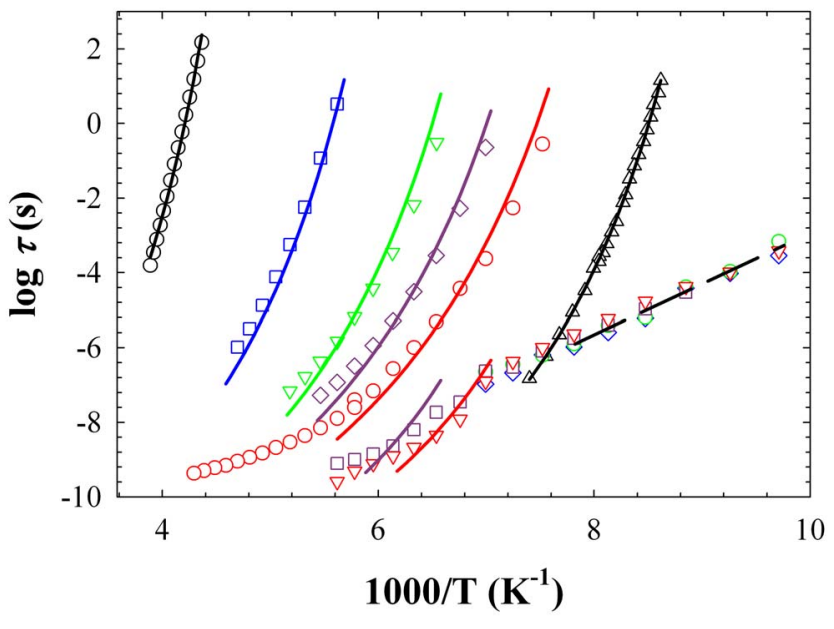

FIG. 4. (Color online) Log plot of the relaxation time vs temperature of all PCB54/toluene mixtures with the following PCB54 wt \%: 70 (blue), 50 (green), 35 (dark pink) and 20 (red); and for pure PCB54 (black circles) and toluene (black triangles). The toluene secondary relaxation is also displayed (dashed black line). Pure PCB54 and toluene relaxational data are taken from Refs. 41 and 42, respectively. The colored and black solid lines are the fits of the AG-SC model to the component dynamics of the mixture and the AG model to pure PCB54 and toluene dynamics, respectively.

fast process displays an Arrhenius-type temperature dependence with a relatively low activation energy $\left(E_{a} / k\right.$ $=1100 \mathrm{~K}$ ). Interestingly, the relaxation time related to this fast component in this temperature range is independent of the PCB54 concentration and, furthermore, coincides with the secondary relaxation of pure toluene which was taken from Ref. 37 and displayed with a dashed black line in Fig. 4.

\section{APPLICATION OF THE AG-SC MODEL}

In the previous section of the manuscript, we have shown that PCB54/toluene mixtures present dynamical heterogeneity, as the relaxational pattern of the mixture is characterized by the presence of two well separated dynamics. As the two components of the mixture are aromatic-and therefore miscible at all compositions-to explain this somewhat intriguing result, one might first invoke some clustering phenomena or concentration fluctuations induced by some preferential interaction among molecules of the same type. However, this scenario seems to be unlikely as both components of the mixture interact via van der Waals forces and clustering phenomena are normally observed in binary mixtures involving at least one liquid forming hydrogen bonds. ${ }^{43} \mathrm{In}$ addition, recent molecular dynamics simulations on mixtures of spheres with large size disparity interacting with a soft potential indicate that this disparity also induces dynamical heterogeneity which is manifested with the presence of two well separated dynamics. ${ }^{44}$

An alternative explanation relies on the concept of cooperativity in glass-forming systems: since the region involved in the cooperative motion giving rise to the glass transition is comparable to molecular dimensions, the local "effective" concentration in this region is different from the macroscopic one and, in particular, it exceeds in the component that is selectively probed. This effect, as mentioned in the Introduc- 
tion known as self-concentration, has been developed by Lodge and McLeish ${ }^{3}$ to describe the component dynamics of miscible polymer blends. Later, its effective soundness and applicability was demonstrated in both dilute miscible polymer blends ${ }^{45}$ and mixtures of low molecular weight glass formers, ${ }^{21}$ as for these systems any effect of clustering or concentration fluctuations can be ruled out.

The concept of self-concentration can be incorporated in the AG theory of the glass transition that relies on the double role of the configurational entropy $\left(S_{c}\right)$, connected to both the relaxation time $(\tau)$ and the size of cooperatively rearranging regions (CRRs) $(\xi) .^{30}$ The AG theory of the glass transition has been intensely disputed along the years. Its merits ${ }^{46}$ and drawbacks ${ }^{47}$ are discussed in recent reviews of the glass transition. Here, we just point out that despite its heuristic nature and the inability to catch the rich and complex dynamics of glass-forming liquids (e.g., Johari-Goldstein secondary relaxations, ${ }^{48}$ dynamical heterogeneity ${ }^{49}$ ), the AG theory is able to properly describe the structural dynamics of a large number of glass-forming liquids, as shown by a large number of experiments ${ }^{50}$ as well as simulations. ${ }^{51}$ While in simulations the configurational entropy is directly provided by the energy landscape, an important approximation of the AG theory applied to experiments is the replacement of the experimentally inaccessible configurational entropy by the entropy of the liquid in excess to the crystal, which can be evaluated through standard calorimetric measurements. However, due to the contribution to the excess entropy related to excess vibrations and secondary relaxation processes, the two magnitudes are never the same. ${ }^{52,53}$ Nevertheless, these two quantities have been shown to be proportional by several studies, ${ }^{54-56}$ at least if nonstructural relaxation related contributions to the excess entropy are absent. ${ }^{57,58}$ Very recent nonlinear dielectric relaxation experiments, allowing the determination of the configurational part of the heat capacity, ${ }^{53}$ confirm the soundness of the proportionality between excess and configurational entropy. ${ }^{59}$ In such a case, the use of the excess entropy instead of the configurational entropy only implies a change in the parameter $C$ of the AG equation [see Eq. (3)].

When applied to bicomponent glass-forming mixtures, the two central equations of the AG theory can be written as $^{11,13,21,22,27-29}$

$$
\tau=\tau_{0}\left(\phi_{\text {eff }}\right) \exp \frac{C\left(\phi_{\text {eff }}\right)}{T S_{c}\left(\phi_{\text {eff }}\right)}
$$

and

$$
\xi / 2=R_{c}\left(\phi_{\mathrm{eff}}\right)=\alpha\left[S_{c}\left(\phi_{\mathrm{eff}}\right)\right]^{-1 / 3},
$$

where $S_{c}, C$, and $\tau_{0}$ in Eq. (3) are respectively the configurational entropy, a temperature independent parameter, and the pre-exponential factor, respectively, $R_{c}$ is the equivalent radius of CRR, and $\alpha$ is a material specific parameter that can be evaluated from the fitting of dynamics data. $\phi_{\text {eff }}$ is the effective concentration that can be related to the selfconcentration through ${ }^{3} \phi_{\text {eff }}=\phi_{s}+\left(1-\phi_{s}\right) \phi$, where $\phi$ is the macroscopic concentration. For low molecular weight glass formers, self-concentration $\phi_{s}$ can be easily related to $R_{c}$ (or $\xi$ ) through simple geometric arguments involving the van der Waals radius of the molecule ${ }^{21}$

$$
R_{c}=R_{\mathrm{vdW}} \phi_{s}^{-1 / 3} \text {. }
$$

A crucial point for the application of the model is the evaluation of the parameters involved in Eq. (3), namely, $C$, $\log \tau_{0}$, and $S_{c}$. These have to be determined in the volume relevant for the relaxation and, therefore, are functions of $\phi_{\text {eff. }}$ According to the approach followed by us, ${ }^{11,13}$ the parameters of Eq. (3) can be calculated assuming a linear combination of the properties of the pure components through the effective concentration in the CRR (for the pre-exponential factor the linear combination is employed for the logarithm). Regarding $\log \tau_{0}$ and $C$, this linear combination is simply an empirical assumption, although it is noteworthy that these two parameters only rarely are very different in a large variety of glass formers. On the other hand, the linear combination of the configurational entropies of the pure components implies that the interaction parameter between PCB54 and toluene is negligible. ${ }^{11}$ As these two systems mainly interact via van der Waals forces, this assumption can be considered reasonable since specific preferential interactions, as in hydrogen bonded liquids, can be excluded.

An important consideration on the use of Eq. (3) with the excess entropy instead of the configurational entropy is that the proportionality constant between the two magnitudes is unlikely to be the same for both components of the mixtures. This means that this proportionality constant will be dependent on the effective concentration in most of the cases and the straightforward employment of the excess entropy instead of the configurational entropy is not rigorously correct. However, for each PCB54/toluene mixture, the effective concentration only varies with temperature by less than 0.1 , as will be shown later in the paper. In this range of variation, the change in the proportionality constant between excess and configurational entropy can always be substantially neglected and, therefore, the excess entropy can still be used instead of the configurational entropy in the AG theory.

The way it has been developed, the AG-SC model relies on the fitting of a single parameter, namely, the factor connecting the size of CRR to the configurational (excess) entropy, as all the other parameters are determined from pure component dynamics and thermodynamic data. In particular, the excess entropy of the pure components can be determined by measuring the heat capacity of pure PCB54 and toluene and integrating the relation $\int_{T_{k}}^{T}\left[\Delta c_{p}(T) / T\right] d T$, where $\Delta c_{p}(T)$ is the excess specific heat: $\Delta c_{p}=c_{p}^{\text {melt }}-c_{p}^{\text {crystal }} \approx c_{p}^{\text {melt }}$ $-c_{p}^{\text {glass }}$. Here, we have employed a linear dependence on the temperature for the excess heat capacity: $\Delta c_{p}(T)=a+b T .^{60}$ The specific heat data for PCB54 and toluene were taken from Refs. 61 and 62, respectively. $T_{K}$ is the Kauzmann temperature, that is, the temperature where the entropy of the liquid equals that of the crystal. Due to the lack of crystalline specific heat data for glass-forming PCB54, the latter quantity was determined by identifying it with the temperature where the relaxation time of the $\alpha$ process tends to diverge. This means that only the structural relaxation related part of the excess entropy is determined in this way. ${ }^{57,58}$ The availability of structural relaxation and specific heat data allows 
TABLE I. Relevant parameters for PCB54 and toluene. Data for the $C$ parameter of the AG relation and for the parameters $a$ and $b$ of the specific heat are referred to a per mole PCB54 and toluene basis for PCB54 and toluene, respectively.

\begin{tabular}{lccccccc}
\hline \hline & $T_{g}(\mathrm{~K})$ & $T_{K}(\mathrm{~K})$ & $C(\mathrm{~kJ} / \mathrm{mol} \mathrm{K})$ & $\log \rho_{0}(\mathrm{~s})$ & $a(\mathrm{~J} / \mathrm{mol} \mathrm{K})$ & $b\left(\mathrm{~J} / \mathrm{mol} \mathrm{K}^{2}\right)$ & $R_{\mathrm{vdW}}(\AA)$ \\
\hline PCB54 & 246 & 192 & 73.5 & -15 & 119 & -0.23 & 3.7 \\
Toluene & 117 & 96 & 73.3 & -14.7 & 105 & -0.31 & 2.9 \\
\hline \hline
\end{tabular}

determining the parameters $C$ and $\log \tau_{0}$ of the pure components of the mixture through the fits of pure components dynamics data via Eq. (3). These fits are shown in Fig. 3 (solid black lines) for both pure PCB54 and toluene. The parameters obtained from the fit of the pure component relaxation data together with the thermodynamic parameters and the van der Waals radii of the two components are all presented in Table I.

Once all the parameters of the pure components are known, Eqs. (3)-(5) can be solved as a function of the effective concentration for each value of $\alpha$. The selected value of $\alpha$ is that providing an effective concentration that, once introduced in Eq. (3), is able to best reproduce the experimental relaxation times. A standard minimization of the mean squared error was carried out to achieve this goal. The solid color lines in Fig. 4 are the best fits of the model to the experimental data. As is possible to observe, the model is able to describe PCB54 related dynamics for relaxation times larger than about $10^{-6}-10^{-7} \mathrm{~s}$. For shorter relaxation times, the model is not able to accurately describe PCB54 related dynamics data. A similar result has been found by Richert and Angell, ${ }^{63}$ who described dynamics data of several low molecular weight glass formers through the AG theory and also found a discrepancy between theory and experimental data at relatively short relaxation times. This suggests that the inability of the AG-SC model to describe dynamics data of bicomponent glass formers for relatively short relaxation times should not be attributed to some limitation of the selfconcentration concept but rather to the intrinsic weakness of the AG theory, with the excess entropy, to describe short time dynamics data. The possible reasons for such a failure must be sought in the inability of the excess entropy to replace the configurational entropy at short relaxation times and/or the inaccuracy of the extrapolation at a relatively high temperature of the specific heat of the glass. Despite this limitation, the AG-SC model with an increasing characteristic length scale when decreasing temperature fits nicely the experimental data in a wide temperature range and, moreover, provides a better description of data than a temperature independent length scale (constant effective concentration). This result is coherent with those found for miscible polymer blends and is discussed in detail for head-to-head polypropylene/ polyisobutylene miscible blend. ${ }^{13}$

Concerning toluene related dynamics, the model has been applied to mixtures with the largest toluene content $(65 \%$ and $80 \%)$ and only in a limited temperature range. The reason is that the AG-SC model is developed to describe equilibrium dynamics, whereas toluene related dielectric response is visible in the available frequency range at temperatures where the slow process related to the relaxation of
PCB54 has fallen out of equilibrium and, therefore, the experimental relaxation times of the low $T_{g}$ component below a certain temperature are affected by the nonequilibrium dynamics imposed by the other component. ${ }^{64}$ This point will be discussed in detail in the next section of the paper. For what concerns the present discussion, we limit the application of the AG-SC model to toluene related dynamics at temperatures for which PCB54 related relaxation shows up in the frequency window available to our experiments. Apart for the low temperature limit marking the crossover from equilibrium to nonequilibrium dynamics, as for PCB54 related dynamics, the AG-SC model could not be applied for very short relaxation times. However, in the case of toluene related dynamics, the shortest relaxation time for which the model could be applied was shorter than in the case of PCB54 related dynamics (about $10^{-9} \mathrm{~s}$ ). These considerations regarding the rather limited range of temperature for the applicability of the AG-SC model to toluene dynamics certainly imply that the results on toluene cooperative length scale are subjected to some degree of approximation. Nonetheless, independent results on toluene dynamics in mixture with other glass formers will be presented in the forthcoming future to confirm and strengthen the present results. ${ }^{65}$

The parameter $\alpha$ of Eq. (4) providing the best fit of data was found to be essentially temperature and concentration independent and its values are $\alpha_{\mathrm{PCB} 54}=25 \pm 1 \mathrm{~J}^{1 / 3}$ $\operatorname{mol}_{\mathrm{PCB} 54}^{-1 / 3} \mathrm{~K}^{-1 / 3}$ and $\alpha_{\text {toluene }}=13 \pm 2 \mathrm{~J}^{1 / 3} \mathrm{~mol}_{\text {toluene }}^{-1 / 3} \mathrm{~K}^{-1 / 3}$. When expressed in consistent mass units, e.g., moles of PCB54, the values of $\alpha$ of the two components of the mixture are not very different from each other: $\alpha_{\text {toluene }}$ $\approx 19 \pm 3 \mathrm{~J}^{1 / 3} \mathrm{~mol}_{\mathrm{PCB} 54}^{-1 / 3} \mathrm{~K}^{-1 / 3}$. This result explains why we were able to fit data with a concentration and temperature independent value of $\alpha$. In a recent paper, we have suggested that a mixing rule for the $\alpha$ parameter of the two components of the mixture should be employed to determine the $\alpha$ parameter at each effective concentration. ${ }^{28}$ In this case, the application of a mixing rule does not provide a strong improvement in the quality of the fits due to the proximity between the $\alpha$ values of the components of the mixture.

The main consequence in using a single value for the $\alpha$ parameter to describe each relaxational process displayed by PCB54/toluene mixtures is that this value can be perceived as the intrinsic $\alpha$ parameter of both PCB54 and toluene. This allows the determination, through Eq. (4), of the characteristic length scale related to the structural relaxation of both pure PCB54 and toluene and in solution. The characteristic length scale associated with PCB54 related dynamics for all investigated mixtures is displayed in Fig. 5 (upper panel) together with the corresponding value of the selfconcentration (lower panel). Despite the significant change 


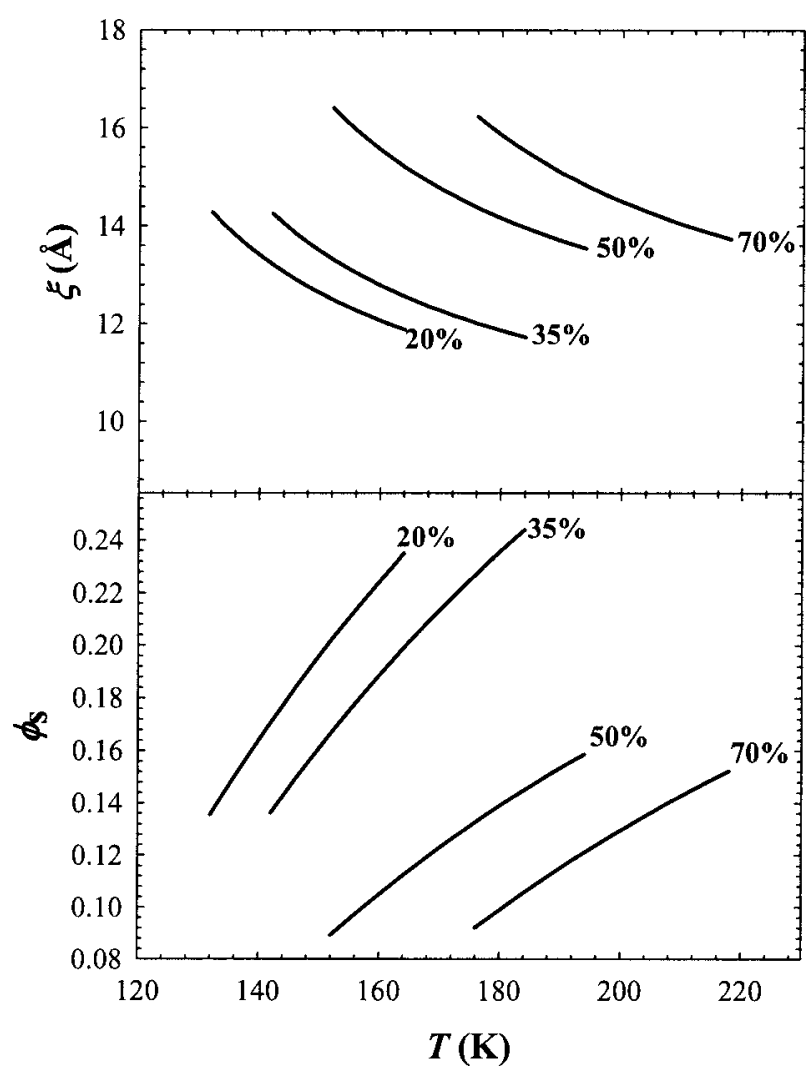

FIG. 5. Temperature dependence of the size of CRR (upper panel) and the self-concentration (lower panel) of PCB54 related dynamics for all investigated mixtures. Percentages refer to PCB54.

in the self-concentration, as this is always rather small, the effective concentration, not shown in the figure, varies by less than 0.1 for all mixtures. As the dynamics of toluene in PCB54 can only be fitted in a tiny temperature range where the temperature variation of the corresponding characteristic length scale is weak, we only report the range of variation of this length scale. This is about $\xi=9-11 \AA$, which corresponds to a value of self-concentration in the range $\phi_{s}=0.2-0.3$, for both mixtures with 65 and $80 \mathrm{wt} . \%$ toluene. From a first inspection of Fig. 5, one can conclude that the increasing length scale imposed by the application of the AG theory [Eq. (4)] results in a mild temperature dependence of this length scale varying by less than $20 \%$ for all mixtures in the available temperature range. Furthermore, the characteristic length increases with PCB54 content, implying that the presence of the relatively more mobile toluene provokes a reduction of the cooperative length scale. Regarding the absolute value of the length scale of both PCB54 and toluene this is comprised between 9 and $17 \AA$, which is of the same order of that found for glass-forming polymers, especially the most flexible ones such as poly(vinyl methyl ether) (PVME) and polyolefins. ${ }^{3-13,22,28}$ On other hand the values of the self-concentration are generally lower than those found for glass-forming polymers but consistent with those found in low molecular weight glass formers $\left(\phi_{s}=0.1-0.3\right.$ at the $T_{g}$ of the mixture). ${ }^{21,66}$ This results is not surprising and can be easily rationalized considering that self-concentration effects are more important for polymers in comparison to low molecular weight glass formers. For the former systems chain

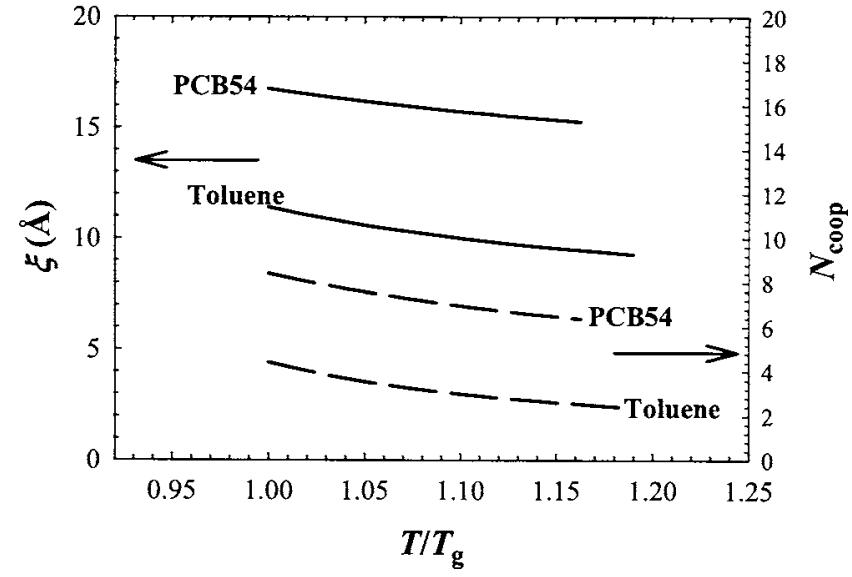

FIG. 6. Size of CRR (solid lines and left axis) and number of structural unit participating to the CRR (dashed lines and right axis) vs the temperature normalized at the $T_{g}$ of pure PCB54 and toluene.

connectivity represents an additional ingredient enhancing self-concentration effects beyond the localized nature of the structural dynamics.

As mentioned previously, the knowledge of the $\alpha$ parameter also allows determining the size of CRR $(\xi)$ and its temperature variation of pure PCB54 and toluene. This is shown in Fig. 6 (left axis) where $\xi$ is plotted vs. the temperature normalized at the $T_{g}$ of each glass former. The number of molecules, coinciding with the basic structural units participating to the cooperative rearrangement due to the rigidity of both PCB54 and toluene molecules, is also shown in the Fig. 6 (right axis). This has been calculated starting from the van der Waals radius, that for PCB54 and toluene equals 3.7 and $2.9 \AA$, respectively, as obtained by employing the approach of Zhao et al. ${ }^{67}$ The number of units participating to the cooperative rearrangement was subsequently calculated considering that part of the volume of CRR is not occupied by van der Waals volume. Thus, the number of units participating to the cooperative rearrangement can be expressed as

$$
N_{\text {coop }}=\frac{\frac{4}{3} \pi\left(\frac{\xi}{2}\right)^{3}}{\nu_{\mathrm{vdw}} \frac{V}{V_{\mathrm{vdw}}}},
$$

where $v_{\mathrm{vdW}}, V_{\mathrm{vdW}}$, and $V$, are the van der Waals volume of one molecule, the molar van der Waals volume, and the molar volume, respectively.

From the observation of Fig. 6, it can be concluded that PCB54 structural relaxation requires larger sizes of CRR compared to the toluene one. This also reflects in the number of basic structural units involved in the cooperative relaxation. This number is of about 8 for PCB54 and 4 for toluene around $T_{g}$.

With our determination of the size of CRR of pure toluene, it is noteworthy that this size bears some relation to the toluene intermolecular distance. In particular, the value of 
the distance between two neighboring toluene molecules is $d \approx 8-9 \AA$, as obtained from the analysis of static structure factor $S(Q)$ measurements. ${ }^{68,69}$ Comparing the value of the size of CRR of toluene at $T_{g}(\xi \approx 11.5 \AA)$ and the intermolecular distance, the former accounts for less than twice the latter implying that the cooperative rearrangement is spatially limited to the first shell around a basic structural unit, in agreement with previous results on several glass-forming polymers. $^{21,22}$ This behavior might be a general feature of glass-forming systems and certainly deserves to be further explored.

Finally, we would like to remark that in our treatment we have implicitly identified the length scale obtained through the effective concentration approach with the size of CRR of the AG theory. This assumption is a priori arbitrary as, within the effective concentration approach, one can only answer the question as to how far is the dynamics of a probe molecule affected by the surrounding. In the way the AG-SC model has been developed, the length scale resulting from the answer to this question is at least proportional to the size of CRR of the AG theory, as we have imposed and subsequently verified the relation between the former length scale and the configurational entropy [Eq. (4)]. In a forthcoming publication, ${ }^{65}$ we will provide arguments suggesting that the length scale deduced from our approach actually coincides with the size of CRR of the AG theory.

\section{OUT-OF-EQUILIBIUM DYNAMICS}

So far, we have centered our attention on the equilibrium dynamics of PCB54/toluene mixtures. In this section, we discuss toluene related dynamics at a relatively low temperature where the system as a whole has fallen out of equilibrium due to the freezing in of PCB54 related relaxation. As observed in Sec. III, the dynamical process under consideration consists of a typical relaxation time with Arrhenius temperature dependence with a relatively low activation energy $\left(E_{a} / k=1100 \mathrm{~K}\right)$. This suggests that the motion related to this process is rather localized in space. In addition, interestingly this process is insensitive to toluene concentration and the related relaxation time is practically equal to that of pure toluene secondary relaxation time, ${ }^{37}$ i.e., the JG relaxation, as shown in Fig. 4. To analyze the nature of this relaxational process in detail, we have compared the dielectric permittivity loss of PCB54/toluene with that of pure toluene taken from Ref. 37. This is shown in Fig. 7 at $118 \mathrm{~K}$. To achieve a straightforward comparison, the dielectric response of PCB54/toluene mixtures has been normalized to $100 \%$ toluene. Considering that the toluene structural relaxation process affects the height of the secondary relaxation peak with its high frequency tail, from the observation of Fig. 7, it is possible to conclude that both the shape and the strength of the relaxational process under consideration are independent of the mixture concentration and practically equal to those of pure toluene. In other words, the localized relaxation observed in PCB54/toluene mixtures bears a strong resemblance with toluene secondary relaxation process as the two processes possess the same shape, strength, and characteristic relaxation time. As toluene is a rigid molecule, the tolu-

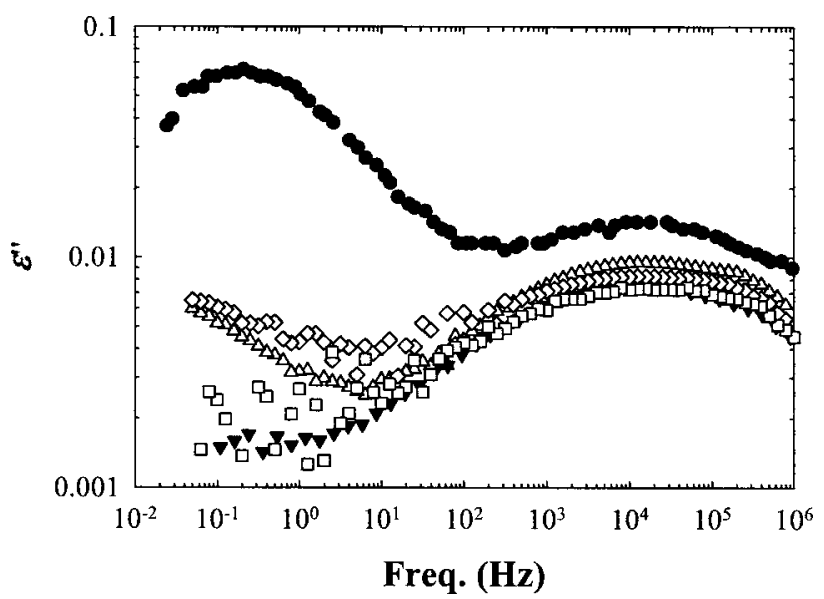

FIG. 7. Loss part of the dielectric permittivity vs frequency at $118 \mathrm{~K}$ of pure toluene (filled circles) taken from Ref. 42 and PCB54/toluene with the following toluene wt \%: 80 (empty up triangles), 65 (filled down triangles), 50 (empty diamonds) and 30 (empty squares).

ene secondary relaxation can be identified with the JG relaxation. This result is rather striking overall when compared to a number of other works, showing that mixing of a glass former with another relatively more rigid glass-former generally provokes a slowing down of the JG secondary relaxation. ${ }^{64,70-72}$ It is worth noticing that some glassforming systems such as PVME also display a secondary relaxation whose shape and characteristic relaxation time are insensitive to the surrounding environment, e.g., polystyrene (PS) as in Refs. 64 and 73. However, in this case such a process is not a $\mathrm{JG}$ relaxation and must be rather related to some intrachain degree of freedom. ${ }^{64,73}$ On the other hand, PVME displays an additional Arrhenius-type relaxational process that, as in other binary mixtures, shows up when mixing PVME with PS and is sensitive to the presence of this other polymer. This suggests that such a process can be considered as the hidden JG process.

The temperature dependence of the JG secondary relaxation has been explained according to the coupling arguments by Capaccioli et al. ${ }^{74}$ However, in the case of toluene, a glass former that lacks internal degree of freedom where the secondary process is certainly a JG relaxation, it is not straightforward to explain the behavior of the secondary relaxation according to the same arguments. In fact, according to such arguments, a slowing down of toluene JG relaxation should be expected. What can be certainly stated is that the out-of equilibrium dynamics induced by density fluctuations, in the case of pure toluene, and by concentration fluctuations, in the case of PCB54/toluene mixtures, gives rise to a restricted motion of toluene moieties which is totally independent of the surrounding environment. Such a restricted motion, strongly resembling the JG relaxation, is also found for highly confined glass formers in nanopores. ${ }^{75,76}$ This analogy suggests that both single and multicomponent glassy systems exhibit localized noncooperative motion, the JG relaxation, that can be perceived as the result of confinement induced by the glassy matrix. 


\section{CONCLUSIONS}

PCB54/toluene mixtures have been investigated by means of broadband dielectric spectroscopy. This system displays dynamical heterogeneity which is manifested with the presence of two relaxational processes. The relatively slow one has been attributed to PCB54 motion in the mixture, whereas the toluene related relaxation is responsible for the relatively fast relaxation. The presence of two well separated dynamics in PCB54/toluene has been rationalized within the framework of the AG-SC model, first introduced to describe the dynamics of miscible polymer blends, incorporating the concept of self-concentration in the AG theory of the glass transition. Apart from the description of equilibrium dynamics data, the model allows the determination of the characteristic length scale for structural relaxation, which is identified with the size of CRR of the AG theory, of both pure PCB54 and toluene in mixture. This has been possible through the knowledge of the glass former specific parameter connecting the characteristic length scale for structural relaxation to the configurational entropy. Sizes of this length scale between 1 and $2 \mathrm{~nm}$ have been found for all systems.

Finally, we have critically discussed the toluene out-ofequilibrium dynamics in mixture with PCB54 and in the pure state. We have found that the relaxational process displayed by both pure toluene and in mixture with PCB54 is insensitive to the surrounding environment since the typical relaxation time, the shape, and the strength of this process are independent of the PCB54 content.

\section{ACKNOWLEDGMENTS}

The authors acknowledge the University of the Basque Country and Basque Country Government (Ref. IT-436-07, Depto. Educación, Universidades e Investigación) and Spanish Minister of Education (MAT 2007-63681) for their support. The support of the European Community within the SoftComp program is also acknowledged.

${ }^{1}$ C. A. Angell, K. L. Ngai, G. B. McKenna, P. F. McMillan, and S. W. Martin, J. Appl. Phys. 88, 3113 (2002).

${ }^{2}$ G. C. Chung, J. A. Kornfield, and S. D. Smith, Macromolecules 27, 964 (1994)

${ }^{3}$ T. P. Lodge and T. C. M. McLeish, Macromolecules 33, 5278 (2000).

${ }^{4}$ B. Min, X. Qiu, M. D. Ediger, M. Pitsikalis, and N. Hadjichristidis, Macromolecules 34, 4466 (2001).

${ }^{5}$ E. Leroy, A. Alegría, and J. Colmenero, Macromolecules 35, 5587 (2002).

${ }^{6}$ Y. Hirose, O. Urakawa, and K. Adachi, Macromolecules 36, 3699 (2003).

${ }^{7}$ J. C. Haley, T. P. Lodge, Y. Y. He, M. D. Ediger, E. D. von Meerwall, and J. Mijovic, Macromolecules 36, 6142 (2003).

${ }^{8}$ Y. He, T. R. Lutz, and M. D. Ediger, J. Chem. Phys. 119, 9956 (2003).

${ }^{9}$ R. Kant, S. K. Kumar, and R. H. Colby, Macromolecules 36, 10087 (2003).

${ }^{10}$ Y. Miwa, Y. Sugino, K. Yamamoto, T. Tanabe, M. Sakaguchi, M. Sakai, and S. Shimada, Macromolecules 37, 6061 (2004).

${ }^{11}$ D. Cangialosi, G. A. Schwartz, A. Alegría, and J. Colmenero, J. Chem. Phys. 123, 144908 (2005).

${ }^{12}$ R. P. Aparicio, A. Arbe, J. Colmenero, B. Frick, L. Willner, D. Richter, and L. J. Fetters, Macromolecules 39, 1060 (2006).

${ }^{13}$ D. Cangialosi, A. Alegría, and J. Colmenero, Macromolecules 39, 448 (2006).

${ }^{14}$ D. Bedrov and G. D. Smith, Macromolecules 39, 8526 (2006).
${ }^{15}$ J. E. G. Lipson and S. T. Milner, J. Polym. Sci., Part B: Polym. Phys. 44, 3528 (2006).

${ }^{16}$ A. F. May and J. K. Maranas, J. Chem. Phys. 125, 024906 (2006).

${ }^{17}$ M. Tyagi, A. Arbe, A. Alegría, J. Colmenero, and B. Frick, Macromolecules 40, 4568 (2007).

${ }^{18}$ W. Zheng and S. L. Simon, J. Polym. Sci., Part B: Polym. Phys. 46, 418 (2007).

${ }^{19}$ R. Lund, L. Willner, A. Alegría, D. Richter, and J. Colmenero, Macromolecules 41, 511 (2008).

${ }^{20}$ J. Colmenero and A. Arbe, Soft Matter 3, 1474 (2007).

${ }^{21}$ D. Cangialosi, A. Alegría, and J. Colmenero, J. Chem. Phys. 126, 204904 (2007).

${ }^{22}$ D. Cangialosi, A. Alegría, and J. Colmenero, Phys. Rev. E 76, 011514 (2007).

${ }^{23}$ T. Blochowicz, C. Karle, A. Kudlik, P. Medick, I. Roggatz, M. Vogel, C. Tschirwitz, J. Wolber, J. Senker, and E. Rossler, J. Phys. Chem. B 103, 4032 (1999).

${ }^{24}$ W. Huang, S. Shariari, and R. Richert, J. Chem. Phys. 123, 164504 (2005).

${ }^{25}$ A. J. Moreno and J. Colmenero, Phys. Rev. E 74, 021409 (2006).

${ }^{26}$ F. Kargl, A. Meyer, M. M. Koza, and H. Schober, Phys. Rev. B 74, 014304 (2006).

${ }^{27}$ G. A. Schwartz, D. Cangialosi, A. Alegría, and J. Colmenero, J. Chem. Phys. 124, 154904 (2006).

${ }^{28}$ D. Cangialosi, A. Alegría, and J. Colmenero, Macromolecules 39, 7149 (2006).

${ }^{29}$ G. A. Schwartz, A. Alegría, and J. Colmenero, J. Chem. Phys. 127, 154907 (2007).

${ }^{30}$ G. Adam and J. H. Gibbs, J. Chem. Phys. 43, 139 (1965).

${ }^{31}$ H. Schlaad and A. H. E. Müller, Macromol. Rapid Commun. 16, 399 (1995).

${ }^{32}$ R. Lund, A. Alegría, L. Goitiandia, J. Colmenero, M. Gonzalez, and P. Lindner, Macromolecules 41, 1364 (2008).

${ }^{33}$ S. Kamath, R. H. Colby, S. K. Kumar, K. Karatasos, G. Floudas, G. Fytas, and J. E. L. Roovers, J. Chem. Phys. 111, 6121 (1999).

${ }^{34}$ Observing the high frequency dielectric response in Fig. 2, it seems that PCB54/toluene mixture with 50 wt \% PCB54 presents a higher dielectric strength than the mixture with 35 wt \% PCB54. However, a detailed analysis of dielectric spectra through the $\mathrm{HN}$ function reveals that the excess loss of the mixture with $50 \mathrm{wt} \%$ PCB54 is due to the high frequency flank of the relatively low frequency process that, in fact, is broader than that of the other mixtures.

${ }^{35}$ K. L. Ngai, P. Lunkenheimer, C. Leon, U. Schneider, R. Brand, and A. C. Loidl, J. Chem. Phys. 115, 1405 (2001).

${ }^{36}$ R. Nozaki, H. Zenitani, A. Minoguchi, and K. Kitai, J. Non-Cryst. Solids 307-310, 349 (2002).

${ }^{37}$ N. B. Olsen, J. Non-Cryst. Solids 235-237, 399 (1998).

${ }^{38}$ C. Hansen, F. Stickel, T. Berger, R. Richert, and E. W. Richert, J. Chem. Phys. 107, 1086 (1997).

${ }^{39}$ S. Havriliak and S. Negami, Polymer 8, 161 (1967).

${ }^{40}$ A. Boersma, J. van Turnhout, and M. Wübbenhorst, Macromolecules 31, 7453 (1998).

${ }^{41}$ R. Casalini and C. M. Roland, Phys. Rev. B 66, 180201 (2002).

${ }^{42}$ A. Doss, G. Hinze, B. Schiener, J. Hemberger, and R. Bohmer, J. Chem. Phys. 107, 1740 (1997); A. Kudlik, S. Benkhof, T. Blochowicz, C. Tschirwitz, and E. Rossler, J. Mol. Liq. 479, 201 (1999).

${ }^{43}$ S. Shahriari, A. Mandanici, L. M. Wang, and R. Richert, J. Chem. Phys. 121, 8960 (2004).

${ }^{44}$ A. J. Moreno and J. Colmenero, Phys. Rev. E 74, 021409 (2006); J. Chem. Phys. 125, 164507 (2006).

${ }^{45}$ T. R. Lutz, Y. Y. He, and M. D. Ediger, Macromolecules 38, 9826 (2005); T. R. Lutz, Y. Y. He, M. D. Ediger, M. Pitsikalis, and N. Hadjichristidis, ibid. 37, 6440 (2004).

${ }^{46}$ P. G. Debenedetti and F. H. Stillinger, Nature (London) 410, 259 (2001); J. Dudowicz, K. F. Freed, and J. F. Douglas, Adv. Chem. Phys. 137, 125 (2008).

${ }^{47}$ J. C. Dyre, Rev. Mod. Phys. 78, 953 (2006).

${ }^{48}$ K. L. Ngai and S. Capaccioli, J. Am. Ceram. Soc. 91, 709 (2008).

${ }^{49}$ R. Richert, J. Phys.: Condens. Matter 14, R703 (2002).

${ }^{50}$ R. Richert and C. A. Angell, J. Chem. Phys. 108, 9016 (1998); D. Prevosto, S. Capaccioli, M. Lucchesi, and P. Rolla, J. Non-Cryst. Solids 351, 2611 (2005); G. A. Schwartz, J. Colmenero, and A. Alegría, Macromolecules 39, 3931 (2006); S. Corezzi, D. Fioretto, and P. Rolla, Nature (London) 420, 653 (2002). 
${ }^{51}$ S. Sastry, Nature (London) 409, 164 (2001); S. Mossa, E. La Nave, H. E. Stanley, C. Donati, F. Sciortino, and P. Tartaglia, Phys. Rev. E 65 041205 (2002).

${ }^{52}$ M. Goldstein, J. Chem. Phys. 64, 4767 (1976); G. P. Johari, ibid. 112, 7518 (2000).

${ }^{53}$ L. M. Wang and R. Richert, Phys. Rev. Lett. 99, 185701 (2007).

${ }^{54}$ C. A. Angell and S. Borick, J. Non-Cryst. Solids 307-310, 393 (2002); C. A. Angell, L.-M. Wang, S. Mossa, Y. Yue, and J. R. D. Copley, AIP Conf. Proc. 708, 473 (2004).

${ }^{55}$ S. Corezzi, L. Comez, and D. Fioretto, Eur. Phys. J. E 14, 143 (2004).

${ }^{56}$ D. Prevosto, S. Capaccioli, M. Lucchesi, D. Leporini, and P. Rolla, J. Phys.: Condens. Matter 16, 6597 (2004).

${ }^{57}$ D. Cangialosi, A. Alegría, and J. Colmenero, Europhys. Lett. 70, 614 (2005).

${ }^{58}$ D. Cangialosi, A. Alegría, and J. Colmenero, J. Chem. Phys. 124, 024906 (2006).

${ }^{59}$ R. Richert, AIP Conf. Proc. (unpublished); private communication (7 April 2008).

${ }^{60}$ I. M. Hodge, Macromolecules 20, 2897 (1987).

${ }^{61}$ C. M. Roland and R. Casalini, J. Therm Anal. Calorim. 83, 87 (2006).

${ }^{62}$ O. Yamamuro, I. Tsukushi, A. Lindqvist, S. Takahara, M. Ishikawa, and T. J. Matsuo, J. Phys. Chem. B 102, 1605 (1998).

${ }^{63}$ R. Richert and C. A. Angell, J. Chem. Phys. 108, 9016 (1998).
${ }^{64}$ C. Lorthioir, A. Alegría, and J. Colmenero, Phys. Rev. E 68, 031805 (2003).

${ }^{65}$ D. Cangialosi, A. Alegría, and J. Colmenero (unpublished).

${ }^{66}$ M. D. Ediger, T. R. Lutz, and Y. Y. He, J. Non-Cryst. Solids 352, 4724 (2006).

${ }^{67}$ Y. H. Zhao, M. H. Abraham, and A. M. Zissimos, J. Org. Chem. 68, $7368(2003)$

${ }^{68}$ A. J. Moreno, A. Alegría, J. Colmenero, M. Prager, H. Grimm, and B. Frick, J. Chem. Phys. 115, 8958 (2001).

${ }^{69}$ C. Alba-Simionesco, G. Dosseh, E. Dumont, B. Frick, B. Geil, D. Morineau, V. Teboul, and Y. Xia, Eur. Phys. J. E 12, 19 (2003).

${ }^{70}$ T. Blochowicz and E. A. Rossler, Phys. Rev. Lett. 92, 225701 (2004).

${ }^{71}$ S. Cerveny, G. A. Schwartz, A. Alegría, R. Bergman, and J. Swenson, J. Chem. Phys. 124, 194501 (2006).

${ }^{72}$ K. Kessairi, S. Capaccioli, D. Prevosto, M. Lucchesi, and P. Rolla, J. Chem. Phys. 127, 174502 (2007).

${ }^{73}$ I. M. Cendoya, A. Alegría, J. Colmenero, H. Grimm, D. Richter, and B. Frick, Macromolecules 32, 4065 (1999).

${ }^{74}$ S. Capaccioli, K. L. Ngai, and N. Shinyashiki, J. Phys. Chem. B 111, 8197 (2007)

${ }^{75}$ A. Schonhals, H. Goering, C. Schick, B. Frick, and R. Zorn, J. NonCryst. Solids 351, 2668 (2005).

${ }^{76}$ K. L. Ngai, J. Phys.: Condens. Matter 11, A119 (1999). 